\title{
The Importance of Knowledge of the Pathophysiology of Drug Hypersensitivity Reactions in Clinical Investigation and Management
}

\section{Zamir Calamita $^{1 *}$, Gabriella Tafuri Missaglia ${ }^{2}$ and Marcela Símaro Gomes ${ }^{2}$}

${ }^{1}$ Medical Immunologist, PhD, Head of the Discipline of Allergy and Immunopathology of the Marília Medical School (Famema), Marília, São Paulo, Brazil

${ }^{2}$ Medical students of Famema, Marília, São Paulo, Brazil

*Corresponding Author: Zamir Calamita, Medical Immunologist, PhD, Head of the Discipline of Allergy and Immunopathology of the Marília Medical School (Famema), Marília, São Paulo, Brazil.

Received: June 24, 2019; Published: July 11, 2019

DOI: 10.31080/ASMS.2019.03.0349

The importance of knowledge of the mechanisms that are involved in drug hypersensitivity reactions (DHR) involves the understanding of its classification itself. In the clinical perspective, it is a challenge to classify the DHR due to countless possibilities of clinical presentations, as well as the large number of available medication. Considering the beginning time of the reactions, we can classify them in two huge groups: immediate DHR, which generally occur in the first hour after being administrated and nonImmediate or delayed DHR, which begins after the first hour of administration [1,2]. The immediate DHR that may occur in the first hour, which beginning may be postponed up to 6 hours, in general, involves IgE mediated hypersensitivity mechanisms (allergic reactions) or non-IgE mediated hypersensitivity mechanisms (non-allergic reactions), also denominated pseudo-allergy. As example of these immediate reactions, we can mention rhinitis, bronchospasm, gastrointestinal disorders (nausea, emesis, cramps and diarrhea), urticaria, angioedema, anaphylaxis, etc. The non- immediate or delayed reactions, which occur subsequently the first hour, usually after days of the drug administration, involve delayed reactions mechanisms described by Gell and Coombs as type II, type III and mainly cells mediated (T cell mediated) type IV hypersensitivity reactions [3]. Examples of non-immediate reactions are exanthematous and eczematous eruptions, erythema multiforme, vasculitis, anemia, leukopenia, thrombocytopenia, renal dysfunction, etc. It is important to say that DHR may involve all types of reactions described by Gell and Coombs, but the most frequent ones involve type I (IgE mediated), type IV (T-cell mediated) hypersensitivity, and immediate non-allergic hypersensitivity. It is important to emphasize the importance of knowing the mechanisms of the DHR, mainly in clinical laboratory research [4-6], since the specific IgE "in vivo" research (skin test), through immediately read or "in vitro" (IgE-specific immunoassay), would be indicated only in DHR IgE mediated cases. Already in the case of the non-IgE immediate reactions, others laboratories exams involving the basophils' activation (commonly not available in clinic practice) could be used [5]. On the other hand, in the late hypersensitivity mediated by $\mathrm{T}$ cells cases, the investigations through late read skin tests or some laboratories exams involving $\mathrm{T}$ cells stimulus (commonly not available in clinic practice) could be indicated [4]. Considering the treatment and focusing in the possibility of specific desensitization to a determinate drug, it is essential the DHR pathophysiology's knowledge $[7,8]$. Another involved aspect in the therapeutic conduction related to the HDR pathophysiology knowledge is the understanding that when dealing with immediate reactions the antihistamines action (often associated with corticosteroids use) is very useful because in immediate reactions it is expected huge histamine liberation by mast cells and basophils. When dealing with late hypersensitivity reactions (T-cell mediated), the need for corticosteroids is frequent, because these reactions, corticosteroids have, at first, a good performance due to their mechanism of action. It is important to say that there are other important possibilities for therapeutic research and conduction, besides numerous complementary measures than those mentioned, primordial in the approach of the RHM, however they were not included in the content of this manuscript. For the purpose of this article was based on only a few simple concepts, we emphasize the importance of knowing 
the pathophysiological mechanisms involved in the triggering of the DHR, so that the health professional can act in a more rational way in the management of such pictures.

\section{Bibliography}

1. Demoly P., et al. "International consensus on drug allergy". Allergy 69 (2014): 420-437.

2. Menezes UP., et al. "Practical aspects in the diagnosis and management of drug hypersensitivity reactions". Brazilian Journal of Allergy and Immunology 2.3 (2014): 91-106.

3. Posadas SJ and Pichler WJ. "Delayed drug hypersensitivity reactions - new concepts". Clinical and Experimental Allergy 37 (2007): 989-999.

4. Mayorga C., et al. "In vitro tests for drug hypersensitivity reactions: an ENDA/EAACI Drug Allergy Interest Group position paper". Allergy 71 (2016): 1103-1134.

5. Viana J., et al. "Estudo da hipersensibilidade a AINES e teste de ativação de basófilos”. Revista da SPDV 73.2 (2015): 293-298.

6. Ensina LF., et al. "Drug hypersensitivity reactions". Rev bras alerg imuno patol 32.2 (2009): 42-47.

7. Sánchez LV., et al. "Drug hypersensitivity and desensitizations: mechanisms and new approaches". International Journal of Molecular Sciences 18 (2017): 1316.

8. Macy E., et al. "Practical management of antibiotic hypersensitivity in 2017". The Journal of Allergy and Clinical Immunology 5 (2017): 577-586.

\section{Volume 3 Issue 8 August 2019}

(C) All rights are reserved by Zamir Calamita., et al. 